Gynäkologe $2011 \cdot 44: 508-508$

DOI 10.1007/s00129-010-2733-z

Online publiziert: 1. Juli 2011

(c) Springer-Verlag 2011

\author{
M.K. Bohlmann · K. Diedrich \\ Klinik für Frauenheilkunde und Geburtshilfe, Universitätsklinikum Schleswig-Holstein, \\ Campus Lübeck
}

\title{
Gerinnungsstörungen in Schwangerschaft und Wochenbett
}

Ein im klinischen Alltag de facto ausschließlich interdisziplinär zu behandelnder Krankheitskomplex betrifft thrombozytäre Gerinnungsstörungen. D. Lüdders hat sich gemeinsam mit Hämostaseologen und Hämatoonkologen der Herausforderung gestellt, die Frage „Maternale Thrombozytopenie und Thrombozytopathie - Was gilt es in der Schwangerschaft zu beachten?" zu beantworten.

Gravidität und Puerperium gelten als risikoreichste Zeiträume im Leben einer Frau, an einer Thrombose zu erkranken. Durch frühzeitige Identifizierung von Risikofaktoren und rechtzeitige präventive Behandlung könnte jedoch ein Großteil der potenziell tödlich verlaufenden Ereignisse verhindert werden. M. Bohlmann hat für Sie die wichtigsten Aspekte internationaler sowie nationaler Leitlinien in Hinblick auf „Prävention und Therapie thromboembolischer Erkrankungen in Schwangerschaft und Wochenbett" zusammengefasst.

Die „Postpartale Hämorrhagie“ stellt auch in Zeiten einer aktiv betreuten Plazentaperiode und potenter Uterotonika eine gefürchtete Geburtskomplikation dar, die ein rechtzeitiges und konsequentes Handeln erfordert. W. Rath präsentiert Ihnen in seiner ausführlichen Übersicht Wege zur Prävention, zum Erkennen und zur situationsadaptierten Behandlung dieser Notfallkonstellation.
Wir hoffen, Ihnen mit dieser Mischung aus verschiedenen „Gerinnungsthemen" ein für Sie interessantes Heft präsentieren zu können.

Herzlichst,

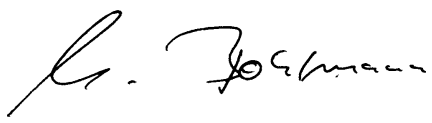

PD Dr. med. M.K. Bohlmann

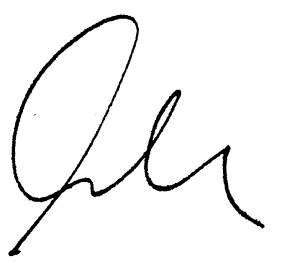

Prof. Dr. med. K. Diedrich

\section{Korrespondenzadresse \\ PD Dr. M.K. Bohlmann}

Klinik für Frauenheilkunde und Geburtshilfe, Universitätsklinikum Schleswig-Holstein, Campus Lübeck

Ratzeburger Allee 160, 23538 Lübeck

michael.bohlmann@uk-sh.de 